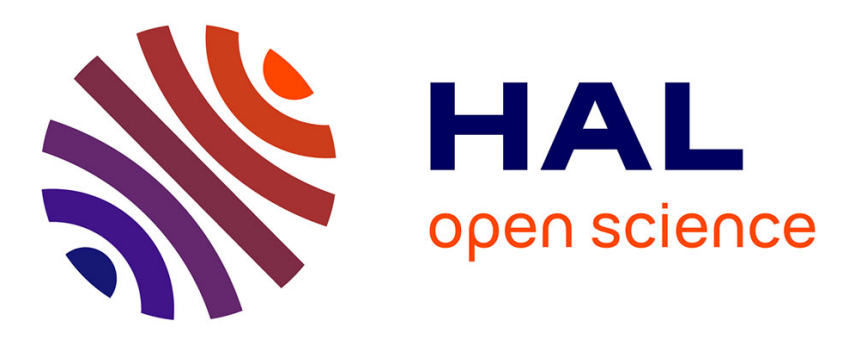

\title{
Linear wavelet estimation in regression with additive and multiplicative noise
}

Christophe Chesneau, Junke Kou, Fabien Navarro

\section{To cite this version:}

Christophe Chesneau, Junke Kou, Fabien Navarro. Linear wavelet estimation in regression with additive and multiplicative noise. Conference of the International Society for Non-Parametric Statistics, Jun 2018, Salerno, Italy. 10.1007/978-3-030-57306-5_13 . hal-01877543v2

\section{HAL Id: hal-01877543 \\ https://hal.science/hal-01877543v2}

Submitted on 14 Feb 2019

HAL is a multi-disciplinary open access archive for the deposit and dissemination of scientific research documents, whether they are published or not. The documents may come from teaching and research institutions in France or abroad, or from public or private research centers.
L'archive ouverte pluridisciplinaire HAL, est destinée au dépôt et à la diffusion de documents scientifiques de niveau recherche, publiés ou non, émanant des établissements d'enseignement et de recherche français ou étrangers, des laboratoires publics ou privés. 


\title{
Linear wavelet estimation in regression with additive and multiplicative noise
}

\author{
Christophe Chesneau, Junke Kou and Fabien Navarro
}

\begin{abstract}
In this paper, we deal with the estimation of an unknown function from a nonparametric regression model with both additive and multiplicative noises. The case of the uniform multiplicative noise is considered. We develop a projection estimator based on wavelets for this problem. We prove that it attains a fast rate of convergence under the mean integrated square error over Besov spaces. A practical extension to automatically select the truncation parameter of this estimator is discussed. A numerical study illustrates the usefulness of this extension.
\end{abstract}

Key words: Nonparametric regression, Multiplicative noise, Rates of convergence, Wavelets

\section{Introduction}

We consider the following unidimensional nonparametric regression model

$$
Y_{i}=U_{i} f\left(X_{i}\right)+V_{i}, \quad i \in\{1, \ldots, n\},
$$

where $f:[0,1] \rightarrow \mathbb{R}$ is an unknown regression function, $X_{1}, \ldots, X_{n}$ are $n$ identically distributed random variables with support on $[0,1], U_{1}, \ldots, U_{n}$ are $n$ identically dis-

\footnotetext{
Christophe Chesneau

Université de Caen - LMNO, FRANCE

e-mail: christophe.chesneau@unicaen.fr

Junke Kou

Guilin University of Electronic Technology, CHINA

e-mail:kjkou@guet.edu.cn

Fabien Navarro (ه)

CREST-ENSAI, BRUZ, FRANCE

e-mail: fabien.navarro@ensai.fr
} 
tributed random variables having the uniform distribution on a symmetric interval around 0 and $V_{1}, \ldots, V_{n}$ are $n$ identically distributed random variables. Moreover, it is supposed that $X_{i}$ and $U_{i}$ are independent, and $U_{i}$ and $V_{i}$ are independent for any $i \in\{1, \ldots, n\}$. Additional technical assumptions on the model will be formulated later. We aim to estimate the unknown function $r:=f^{2}$ from $\left(X_{1}, Y_{1}\right), \ldots,\left(X_{n}, Y_{n}\right)$; the random vectors $\left(U_{1}, V_{1}\right), \ldots,\left(U_{n}, V_{n}\right)$ form the multiplicative-additive noise. The model (1) can be viewed as a natural extension of the standard nonparametric regression model; the main novelty is the presence of a multiplicative uniform noise that perturbed the unknown function $f$. Such multiplicative regression model as (1) is very popular in various application areas, particularly in signal processing (e.g. for Global Positioning System (GPS) signal detection in which not only additive noise but also multiplicative noise is encountered Huang et al. (2013)), or in econometrics (e.g. for volatility estimation where the source of noise is multiplicative Härdle and Tsybakov (1997), also for deterministic and stochastic frontier estimation where the noise is multiplicative and both multiplicative and additive respectively Simar and Wilson (2000)). On the other hand, let us mention that some connexions exist with the so called heteroscedastic nonparametric regression model. See, for instance, Chichignoud (2012), Comte (2015) and Cai et al. (2008). In particular, Cai et al. (2008) consider the estimation of $r$ in the heteroscedastic nonparametric regression model defined as (1) with $X_{1}$ deterministic, $V_{1}$ deterministic but unknown (it is an unknown function of $X_{1}$ ) and general assumptions on $U_{1}$. The form of the model is the same but the intrinsic definition is different. In this paper, we propose to estimate $r$ with wavelet methods. Such methods have the advantage to capture the possible complexity of this unknown function. A natural linear wavelet estimator is then developed. With a suitable choice of a tuning parameter inherent of this estimator, we prove that it attains a fast rate of convergence under the mean integrated square error over Besov spaces. One drawback of this estimator is that the theoretical choice for the tuning parameter depends on a supposed unknown smoothness of $r$. We then provide practical solution to this problem to choose the truncation level of our linear wavelet estimator using an adapted version of the 2-Fold Cross Validation (2FCV) method introduced by Nason Nason (1996). A numerical study is performed to show the applicability of this extension.

The rest of this paper is organized as follows. In Section 2, we briefly present basics on wavelets and Besov balls. Additional assumptions on the model (1), the considered wavelet estimator and the main result are given in Section 3. Section 4 is devoted to the simulation study. The technical details for the proof of our main result are postponed in Section 6.

\section{Basics on wavelets and Besov balls}

For the purpose of this paper, we use the compactly supported wavelets of the Daubechies family. We present the essential below, all the details can be found in, e.g., Daubechies (1992) and Mallat (2008). For any $j \geq 0$, we set $\Lambda_{j}=\left\{0, \ldots, 2^{j}-\right.$ 
$1\}$ and, for $k \in \Lambda_{j}$,

$$
\phi_{j, k}(x)=2^{j / 2} \phi\left(2^{j} x-k\right), \quad \psi_{j, k}(x)=2^{j / 2} \psi\left(2^{j} x-k\right) .
$$

Following the methodology of Cohen et al. (1993), there exists an integer $\tau$ such that, for any integer $j_{0} \geq \tau$, the collection of functions

$$
\mathscr{S}=\left\{\phi_{j_{0}, k}, k \in \Lambda_{j_{0}} ; \psi_{j, k} ; j \in \mathbb{N}-\left\{0, \ldots, j_{0}-1\right\}, k \in \Lambda_{j}\right\}
$$

forms an orthonormal basis of $\mathbb{L}^{2}([0,1])$. Therefore, for any integer $j_{0} \geq \tau$ and $h \in$ $\mathbb{L}^{2}([0,1])$, we have the following wavelet expansion:

$$
h(x)=\sum_{k \in \Lambda_{j_{0}}} \alpha_{j_{0}, k} \phi_{j_{0}, k}(x)+\sum_{j=j_{0}}^{\infty} \sum_{k \in \Lambda_{j}} \beta_{j, k} \psi_{j, k}(x), \quad x \in[0,1],
$$

where

$$
\alpha_{j_{0}, k}=\int_{0}^{1} h(x) \phi_{j_{0}, k}(x) d x, \quad \beta_{j, k}=\int_{0}^{1} h(x) \psi_{j, k}(x) d x,
$$

Also, let us mention that $\int_{0}^{1} \phi_{j, k}(x) d x=2^{-j / 2}$, which will be a crucial technical point in the proof. Let $P_{j}$ be the orthogonal projection operator from $L^{2}([0,1])$ onto the space $V_{j}$ with the orthonormal basis $\left\{\phi_{j, k}(\cdot)=2^{j / 2} \phi\left(2^{j} \cdot-k\right), k \in \Lambda_{j}\right\}$. Then, for any $h \in L^{2}([0,1])$, we have

$$
P_{j} h(x)=\sum_{k \in \Lambda_{j}} \alpha_{j, k} \phi_{j, k}(x), \quad x \in[0,1] .
$$

Besov spaces have the feature to capture a wide variety of smoothness properties in a function including spatially inhomogeneous behavior, see Härdle et al. (2012); Meyer (1992); Triebel (1994) for further details. Definitions of those spaces are given below. Suppose that $\phi$ is $m$ regular (i.e. $\phi \in C^{m}$ and $\left|D^{\alpha} \phi(x)\right| \leq c\left(1+|x|^{2}\right)^{-l}$ for each $l \in \mathbb{Z}$, with $\alpha=0,1, \ldots, m)$. Let $h \in L^{p}([0,1]), p, q \in[1, \infty]$ and $0<s<m$. Then the following assertions are equivalent:

(1) $h \in B_{p, q}^{s}([0,1])$; (2) $\left\{2^{j s}\left\|P_{j+1} h-P_{j} h\right\|_{p}\right\} \in l_{q}$; (3) $\left\{2^{j\left(s-\frac{1}{p}+\frac{1}{2}\right)}\left\|\beta_{j, .}\right\|_{p}\right\} \in l_{q}$. The Besov norm of $h$ can be defined by

$$
\|h\|_{B_{p, q}^{s}}:=\left\|\left(\alpha_{\tau, .}\right)\right\|_{p}+\left\|\left(2^{j\left(s-\frac{1}{p}+\frac{1}{2}\right)}\left\|\beta_{j, .}\right\|_{p}\right)_{j \geq \tau}\right\|_{q}, \text { where }\left\|\beta_{j, .}\right\|_{p}^{p}=\sum_{\mathbf{k} \in \Lambda_{j}}\left|\beta_{j, k}\right|^{p} .
$$

\section{Assumptions, estimators and main result}

Technical assumptions on the model (1) are formulated below.

A.1 We suppose that $f:[0,1] \rightarrow \mathbb{R}$ is bounded from above.

A.2 We suppose that $X_{1} \sim \mathscr{U}([0,1])$. 
A.3 We suppose that $U_{1} \sim \mathscr{U}([-\theta, \theta])$ with $\theta>0$ a fixed real number.

A.4 We suppose that $V_{1}$ has a moment of order 4 .

A.5 We suppose that $X_{i}$ and $V_{i}$ are independent for any $i \in\{1, \ldots, n\}$.

Let us observe that A.2 specifies that we consider a uniform design and that $\mathbf{A . 3}$ specifies that the uniform multiplicative noise is considered over a symmetric interval around 0. The assumption A.5 implies that $V_{i}$ is not a function of $X_{i}$ a fortiori.

We construct our linear wavelet estimators for $r$ as follows:

$$
\hat{r}_{j_{0}, n}(x):=\sum_{k \in \Lambda_{j_{0}}} \hat{\alpha}_{j_{0}, \mathbf{k}} \phi_{j_{0}, k}(x), \quad x \in[0,1],
$$

where

$$
\hat{\alpha}_{j, k}:=\frac{3}{\theta^{2}}\left(\frac{1}{n} \sum_{i=1}^{n} Y_{i}^{2} \phi_{j, k}\left(X_{i}\right)-\mathbb{E}\left(V_{1}^{2}\right) 2^{-j / 2}\right) .
$$

The definition of $\hat{\alpha}_{j, k}$ rests on technical consideration which will be presented later. In spite of the simplicity of its construction, its performances strongly depend on the choice of level $j_{0}$. Further details on the linear wavelet estimator in a standard nonparametric regression setting can be found in Härdle et al. (2012). Recent developments can be found in Chaubey et al. (2015).

The following result determines the rates of convergence attained by $\hat{r}_{j_{0}, n}$ via the MISE over Besov spaces.

Proposition 1. Consider the problem defined by (1) under the assumptions A.1-A.5, let $r \in B_{p, q}^{s}([0,1])$ with $p, q \in[1, \infty), s>1 / p$. Then the linear wavelet estimator $\hat{r}_{j_{0}, n}$ with $2^{j_{*}} \sim n^{\frac{1}{2 s^{\prime}+1}}$ and $s^{\prime}=s-(1 / p-1 / 2)_{+}$satisfies

$$
\mathbb{E}\left[\int_{0}^{1}\left(\hat{r}_{j_{0}, n}(x)-r(x)\right)^{2} d x\right] \lesssim n^{-\frac{2 s^{\prime}}{2 s^{\prime}+1}} .
$$

The level $j_{0}$ as defined in Proposition 1 is chosen to minimize as possible the MISE of $\hat{r}_{j_{0}, n}$ over Besov spaces. The rate of convergence $n^{-\frac{2 s^{\prime}}{2 s^{\prime}+1}}$ is not a surprise ; it generally corresponds to the one obtained in the standard nonparametric regression estimation. See Härdle et al. (2012), Tsybakov (2009) and Comte (2015). The proof of Proposition 1 is based on a suitable decomposition of the MISE and some intermediary results on the probabilistic properties of the wavelet coefficient estimator (3) (see Lemmas 1 and 2 in Section 6). The rest of this section is devoted to the practical aspect of the estimator (2), with alternatives on the choice of the level $j_{0}$. In particular, we propose a candidate by adapting version of the 2-Fold Cross Validation (2FCV) method originally developed by Nason for choosing the threshold parameter in wavelet shrinkage Nason (1996). 


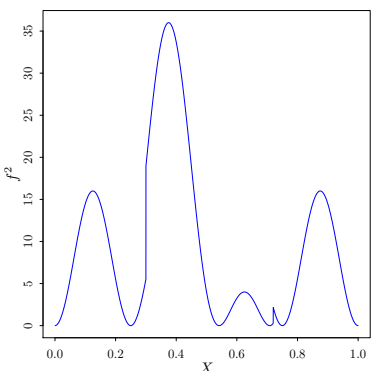

(a) HeaviSine

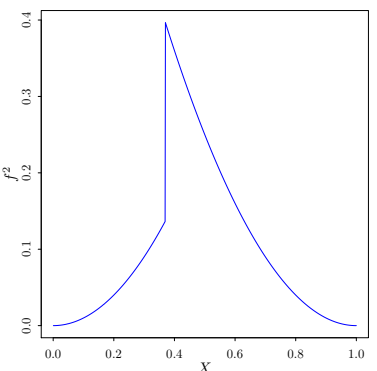

(b) Ramp

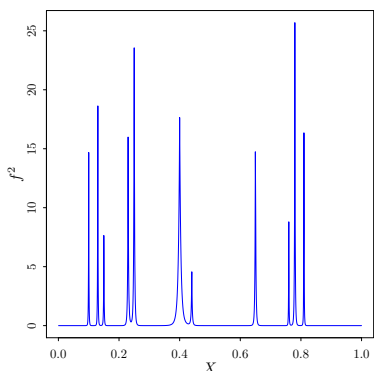

(c) Bumps

Fig. 1 (a)-(c): The three test (squared) functions to be estimated.

\section{Simulation study}

In order to illustrate the empirical performance of the proposed estimator, a numerical illustration was produced. In order to set in a realistic context, we proposed to use an automatic selection method of the estimator truncation parameter (not depending on the regularity of the function to be estimated). Simulations were performed using $\mathrm{R}$ and in particular the rwavelet package Navarro and Chesneau (2018), available from https://github.com/fabnavarro/rwavelet.

The simulated data were generated according to (1), where $n=4096, X_{i}$ 's are uniformly distributed on $[0,1], U_{i}$ 's are $\mathscr{U}([-1,1])($ so $\theta=1)$ and $V_{i}$ are $\mathscr{N}\left(0, \sigma^{2}\right)$ variables and independent of $X_{i}$ 's with $\sigma^{2}=0.01$. Daubechies' compactly-supported wavelet with 8 vanishing moments were used. We consider three standard test functions for $f$, commonly used in the wavelet literature (HeaviSine, Ramp and Bumps, see Donoho et al. (1995)). Recall that we wish to estimate $r=f^{2}$. The squared version of those functions are plotted in Figure 1.

In the case of fixed design, the calculation of wavelet-based estimators is simple and fast, thanks to Mallat's pyramidal algorithm (Mallat (2008)). In the case of uniform random design, the implementation requires some changes and several strategies have been developed in the literature (see e.g. Cai et al. (1998); Hall et al. (1997)). For uniform design regression, Cai and Brown (1999) proposed to use and studied an approach in which the wavelet coefficients are computed by a simple application of Mallat's algorithm using the ordered $Y_{i}$ 's as input variables. We have followed this approach because it preserves the simplicity of calculation and the efficiency of the equispaced algorithm. In the context of wavelet regression in random design with heteroscedastic noise, Navarro and Saumard (2017b) and Kulik et al. (2009) also adopted this approach. Nason adjusted the usual 2FCV method to choose the threshold parameter in wavelet shrinkage (see Nason (1996)). His strategy was used for the selection of linear wavelet estimators by Navarro and Saumard (2017b). We have chosen to use this approach to select the truncation parameter $j_{0}$ of the linear estimator $\hat{r}_{j_{0}, n}$. More precisely, we built a collection of linear estimators $\hat{r}_{j_{0}, n}, j_{0}=0,1, \ldots, \log 2(n)-1$ (by successively adding whole resolution levels 
of wavelet coefficients), and select the best among this collection by minimizing a $2 \mathrm{FCV}$ criterion denoted by $2 \mathrm{FCV}\left(j_{0}\right)$. The resulting estimator of the truncation level is denoted by $\hat{j}_{0}$ and the corresponding estimator of $r$ by $\hat{r}_{\hat{j}_{0}, n}$ (see Navarro and Saumard (2017b,a) for more details).

For a single experiment, and for each of the three test functions, with a sample size $n=4096$, we display the observations and the unknown function $r$ in Figure 2(a). A sample of three estimators from the collection is also shown in the Figure 2(b). Graphs of the curves associated with the selection criterion (i.e. $\left.2 \mathrm{FCV}\left(j_{0}\right)\right)$ are also displayed in Figure 2(c). In order to be able to evaluate the performance of this criterion, the Mean Square Error curves (i.e. $\operatorname{MSE}\left(\hat{r}_{j_{0}, n}, r\right)=$ $\left.\left.\frac{1}{n} \sum_{i=1}^{n}\left(r\left(X_{i}\right)-\hat{r}_{j_{0}, n}\left(X_{i}\right)\right)^{2}\right)\right)$ are also shown (in blue). We denote by $j_{0}^{*}$, the parameter selected by minimizing this quantity. It can be observed that $2 \mathrm{FCV}\left(j_{0}\right)$ gives very reliable estimate for the $\operatorname{MSE}\left(\hat{r}_{j_{0}, n}, r\right)$, and in turn, also a high-quality estimate of the optimal model. Indeed, in this case, the method allows to find the oracle of the collection (i.e. that obtained by assuming the regularity of the function to be estimated known) for the three signals.

\section{Conclusion}

In this paper, we develop a simple wavelet methodology for the problem of estimating an unknown function subject to additive and multiplicative noises. Focusing on a uniform multiplicative noise, we construct a linear wavelet estimator that attains a fast rate of convergence. Then some extensions of the estimator are presented, with a numerical study showing the usefulness of the method.

A possible extension of this work would be to consider a more general model with $\theta$ unknown for instance or a more general assumption on the distribution of the multiplicative noise. Another possible extension would be to construct another wavelet estimation procedure involving thresholding of the wavelet coefficient estimators and also dependence on the observations, as in Chesneau et al. (2015) for the additive noise only. These aspects need further investigations that we leave for a future work.

\section{Proofs}

To prove Proposition 1, we use the following two lemmas.

Lemma 1. Let $j \geq \tau, k \in \Lambda_{j}, \hat{\alpha}_{j, k}$ be (3). Then, under A.1-A.5, we have

$$
\mathbb{E}\left[\hat{\alpha}_{j, k}\right]=\alpha_{j, k} .
$$

Proof of Lemma 1. Using the independence assumptions on the random variables, A.1-A.5 with $\mathbb{E}\left[U_{1}\right]=0$, observe that 

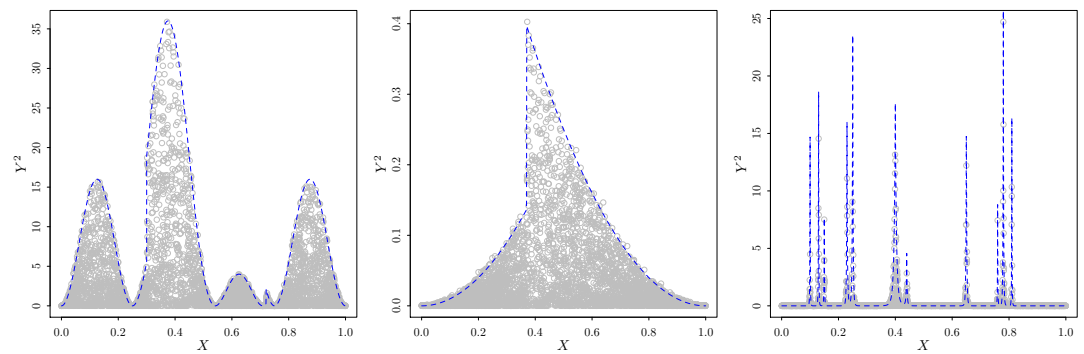

(a)
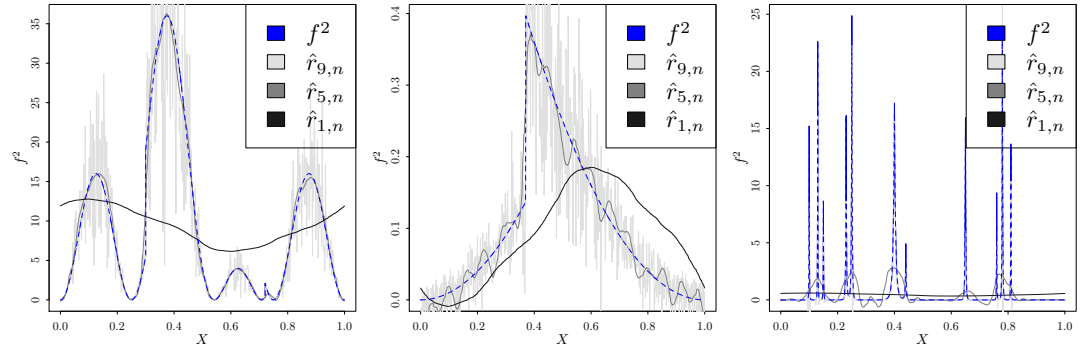

(b)
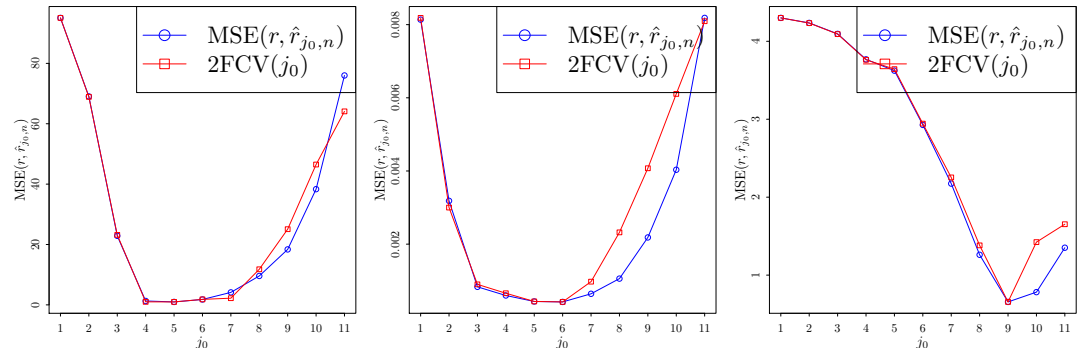

(c)
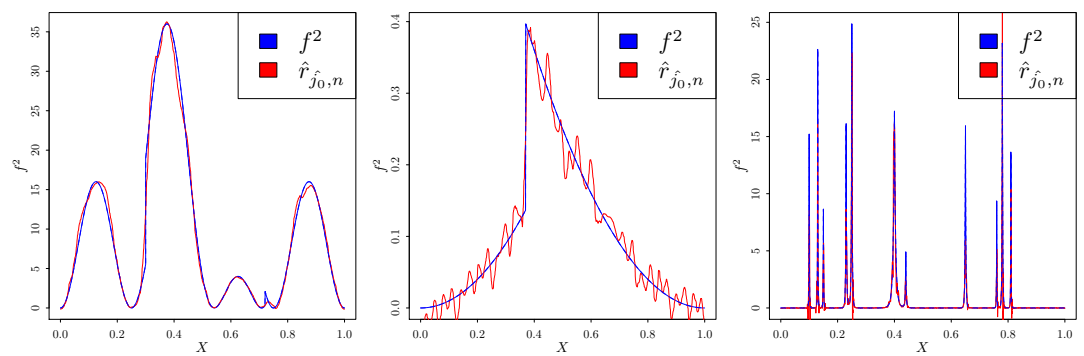

(d)

Fig. 2 (a): Noisy observations $\left(X, Y^{2}\right)$. (b): Sample of the model collection. (c): Graph of the MSE (blue) against $j_{0}$ and (re-scaled) $2 \mathrm{FCV}$ criterion. (d): Typical estimations from one simulation with $n=4096$. Blue lines indicate the true functions, red lines correspond to the estimators $\hat{r}_{\hat{j}_{0}, n}$. 


$$
\mathbb{E}\left[U_{1} V_{1} f\left(X_{1}\right) \phi_{j, k}\left(X_{1}\right)\right]=\mathbb{E}\left[U_{1}\right] \mathbb{E}\left[V_{1}\right] \mathbb{E}\left[f\left(X_{1}\right) \phi_{j, k}\left(X_{1}\right)\right]=0
$$

and

$$
\mathbb{E}\left[V_{1}^{2} \phi_{j, k}\left(X_{1}\right)\right]=\mathbb{E}\left[V_{1}^{2}\right] \mathbb{E}\left[\phi_{j, k}\left(X_{1}\right)\right]=\mathbb{E}\left[V_{1}^{2}\right] \int_{0}^{1} \phi_{j, k}(x) d x=\mathbb{E}\left[V_{1}^{2}\right] 2^{-j / 2} .
$$

Therefore, using similar mathematical arguments with $\mathbb{E}\left[U_{1}^{2}\right]=\frac{\theta^{2}}{3}$, we have

$$
\begin{aligned}
\mathbb{E}\left[\hat{\alpha}_{j, k}\right] & =\frac{3}{\theta^{2}} \mathbb{E}\left[\frac{1}{n} \sum_{i=1}^{n} Y_{i}^{2} \phi_{j, k}\left(X_{i}\right)-\mathbb{E}\left[V_{1}^{2}\right] 2^{-j / 2}\right] \\
& =\frac{3}{\theta^{2}}\left(\mathbb{E}\left[Y_{1}^{2} \phi_{j, k}\left(X_{1}\right)\right]-\mathbb{E}\left[V_{1}^{2}\right] 2^{-j / 2}\right) \\
& =\frac{3}{\theta^{2}}\left(\mathbb{E}\left[U_{1}^{2} r\left(X_{1}\right) \phi_{j, k}\left(X_{1}\right)\right]+2 \mathbb{E}\left[U_{1} V_{1} f\left(X_{1}\right) \phi_{j, k}\left(X_{1}\right)\right]+\mathbb{E}\left[V_{1}^{2} \phi_{j, k}\left(X_{1}\right)\right]\right. \\
& =\frac{3}{\theta^{2}} \mathbb{E}\left[U_{1}^{2}\right] \mathbb{E}\left[r\left(V_{1}^{2} \phi_{j, k}\left(X_{1}\right)\right]\right)
\end{aligned}
$$

Lemma 1 is proved.

Lemma 2. Let $j \geq \tau$ such that $2^{j} \leq n, k \in \Lambda_{j}, \hat{\alpha}_{j, k}$ be (3). Then, under (A.A.1)(A.A.5),

$$
\mathbb{E}\left[\left(\hat{\alpha}_{j, k}-\alpha_{j, k}\right)^{2}\right] \lesssim \frac{1}{n}
$$

Proof of Lemma 2. Owing to Lemma 1 we have $\mathbb{E}\left[\hat{\alpha}_{j, k}\right]=\alpha_{j, k}$. Therefore

$$
\begin{aligned}
\mathbb{E}\left[\left(\hat{\alpha}_{j, k}-\alpha_{j, k}\right)^{2}\right] & =\operatorname{Var}\left[\hat{\alpha}_{j, k}\right]=\frac{9}{\theta^{4}} \operatorname{Var}\left[\frac{1}{n} \sum_{i=1}^{n} Y_{i}^{2} \phi_{j, k}\left(X_{i}\right)-\mathbb{E}\left[V_{1}^{2}\right] 2^{-j / 2}\right] \\
& =\frac{9}{\theta^{4}} \operatorname{Var}\left[\frac{1}{n} \sum_{i=1}^{n} Y_{i}^{2} \phi_{j, k}\left(X_{i}\right)\right] \\
& =\frac{9}{\theta^{4}} \frac{1}{n} \operatorname{Var}\left[Y_{1}^{2} \phi_{j, k}\left(X_{1}\right)\right] \lesssim \frac{1}{n} E\left[Y_{1}^{4} \phi_{j, k}^{2}\left(X_{1}\right)\right] \\
& \lesssim \frac{1}{n}\left[\mathbb{E}\left[U_{1}^{4} f^{4}\left(X_{1}\right) \phi_{j, k}^{2}\left(X_{1}\right)\right]+\mathbb{E}\left[V_{1}^{4} \phi_{j, k}^{2}\left(X_{1}\right)\right]\right] \\
& =\frac{1}{n}\left[\mathbb{E}\left[U_{1}^{4}\right] \mathbb{E}\left[f^{4}\left(X_{1}\right) \phi_{j, k}^{2}\left(X_{1}\right)\right]+\mathbb{E}\left[V_{1}^{4} \phi_{j, k}^{2}\left(X_{1}\right)\right]\right] .
\end{aligned}
$$

By A.1 and $\mathbb{E}\left[\phi_{j, k}^{2}\left(X_{1}\right)\right]=\int_{0}^{1} \phi_{j, k}^{2}(x) d x=1$, we have $\mathbb{E}\left[f^{4}\left(X_{1}\right) \phi_{j, k}^{2}\left(X_{1}\right)\right] \lesssim 1$. On the other hand, by A.4 and A.5, we have

$$
\mathbb{E}\left[V_{1}^{4} \phi_{j, k}^{2}\left(X_{1}\right)\right]=\mathbb{E}\left[V_{1}^{4}\right] \mathbb{E}\left[\phi_{j, k}^{2}\left(X_{1}\right)\right]=\mathbb{E}\left[V_{1}^{4}\right] \lesssim 1
$$


Thus all the terms in the brackets of (4) are bounded from above. This ends the proof of Lemma 2.

Proof of Proposition 1 from Lemmas 1 and 2. The main lines of the proof use standard arguments (see, for instance, Härdle et al. (2012)). The key result remains Lemma 2 above and a suitable choice for $j_{0}$ which balance the biais and the rest term of term. More precisely, by the definition of projector, we have

$$
\mathbb{E}\left[\int_{0}^{1}\left|\hat{r}_{j_{0}, n}(x)-r(x)\right|^{2} d x\right]=\mathbb{E}\left[\left\|\hat{r}_{j_{0}, n}-P_{j_{*}} r\right\|_{2}^{2}\right]+\left\|P_{j_{*}} r-r\right\|_{2}^{2} .
$$

The orthonormality of the wavelet basis gives

$$
\mathbb{E}\left[\left\|\hat{r}_{j_{0}, n}-P_{j_{*}} r\right\|_{2}^{2}\right]=\mathbb{E}\left[\left\|\sum_{k \in \Lambda_{j_{*}}}\left(\hat{\alpha}_{j_{*}, k}-\alpha_{j_{*}, k}\right) \phi_{j_{*}, k}\right\|_{2}^{2}\right]=\sum_{k \in \Lambda_{j_{*}}} \mathbb{E}\left[\left(\hat{\alpha}_{j_{*}, k}-\alpha_{j_{*}, k}\right)^{2}\right] .
$$

According to Lemma 2, $\left|\Lambda_{j_{*}}\right| \sim 2^{j_{*}}$ and $2^{j_{*}} \sim n^{\frac{1}{2 s^{\prime}+1}}$,

$$
\mathbb{E}\left[\left\|\hat{r}_{j_{0}, n}-P_{j_{*}} r\right\|_{2}^{2}\right] \lesssim \frac{2^{j_{0}}}{n} \lesssim n^{-\frac{2 s^{\prime}}{2 s^{\prime}+1}} .
$$

When $p \geq 2, s^{\prime}=s$. By Hölder inequality and $r \in B_{p, q}^{s}([0,1])$,

$$
\left\|P_{j_{0}} r-r\right\|_{2}^{2} \lesssim\left\|P_{j_{0}} r-r\right\|_{p}^{2} \lesssim 2^{-2 j_{0} s} \lesssim n^{-\frac{2 s}{2 s+\mathrm{I}}}
$$

When $1 \leq p<2$ and $s>1 / p, B_{p, q}^{s}([0,1]) \subseteq B_{2, \infty}^{s^{\prime}}([0,1])$

$$
\left\|P_{j_{0}} r-r\right\|_{2}^{2} \lesssim \sum_{j=j_{0}}^{\infty} 2^{-2 j s^{\prime}} \lesssim 2^{-2 j j_{0} s^{\prime}} \lesssim n^{-\frac{2 s^{\prime}}{2 s^{\prime}+1}}
$$

Therefore, in both cases,

$$
\left\|P_{j_{0}} r-r\right\|_{2}^{2} \lesssim n^{-\frac{2 s^{\prime}}{2 s^{\prime}+1}} .
$$

By (5), (6) and (7), we obtain

$$
\mathbb{E}\left[\int_{0}^{1}\left|\hat{r}_{j, n}(x)-r(x)\right|^{2} d x\right] \lesssim n^{-\frac{2 s^{\prime}}{2 s^{\prime}+1}} .
$$

Proposition 1 is proved. 


\section{References}

Cai, T. T. and L. D. Brown (1999). Wavelet estimation for samples with random uniform design. Statistics \& probability letters 42(3), 313-321.

Cai, T. T., L. D. Brown, et al. (1998). Wavelet shrinkage for nonequispaced samples. The Annals of Statistics 26(5), 1783-1799.

Cai, T. T., L. Wang, et al. (2008). Adaptive variance function estimation in heteroscedastic nonparametric regression. The Annals of Statistics 36(5), 20252054.

Chaubey, Y. P., C. Chesneau, and H. Doosti (2015). Adaptive wavelet estimation of a density from mixtures under multiplicative censoring. Statistics 49(3), 638-659.

Chesneau, C., J. Fadili, and B. Maillot (2015). Adaptive estimation of an additive regression function from weakly dependent data. Journal of Multivariate Analysis 133, 77-94.

Chichignoud, M. (2012). Minimax and minimax adaptive estimation in multiplicative regression: locally bayesian approach. Probability Theory and Related Fields 153(3-4), 543-586.

Cohen, A., I. Daubechies, and P. Vial (1993). Wavelets on the interval and fast wavelet transforms. Applied and computational harmonic analysis.

Comte, F. (2015). Estimation non-paramétrique. Spartacus-IDH.

Daubechies, I. (1992). Ten lectures on wavelets, Volume 61. Siam.

Donoho, D. L., I. M. Johnstone, G. Kerkyacharian, and D. Picard (1995). Wavelet shrinkage: asymptopia? Journal of the Royal Statistical Society. Series B (Methodological), 301-369.

Hall, P., B. A. Turlach, et al. (1997). Interpolation methods for nonlinear wavelet regression with irregularly spaced design. The Annals of Statistics 25(5), 19121925.

Härdle, W., G. Kerkyacharian, D. Picard, and A. Tsybakov (2012). Wavelets, approximation, and statistical applications, Volume 129. Springer Science \& Business Media.

Härdle, W. and A. Tsybakov (1997). Local polynomial estimators of the volatility function in nonparametric autoregression. Journal of econometrics 81(1), 223242.

Huang, P., Y. Pi, and I. Progri (2013). Gps signal detection under multiplicative and additive noise. The Journal of Navigation 66(4), 479-500.

Kulik, R., M. Raimondo, et al. (2009). Wavelet regression in random design with heteroscedastic dependent errors. The Annals of Statistics 37(6A), 3396-3430.

Mallat, S. (2008). A wavelet tour of signal processing: the sparse way. Academic press.

Meyer, Y. (1992). Wavelets and operators, Volume 1. Cambridge university press.

Nason, G. P. (1996). Wavelet shrinkage using cross-validation. Journal of the Royal Statistical Society. Series B (Methodological), 463-479.

Navarro, F. and C. Chesneau (2018). R package rwavelet: Wavelet Analysis. (Version 0.1 .0 ). 
Navarro, F. and A. Saumard (2017a). Efficiency of the v-fold model selection for localized bases.

Navarro, F. and A. Saumard (2017b). Slope heuristics and v-fold model selection in heteroscedastic regression using strongly localized bases. ESAIM: Probability and Statistics 21, 412-451.

Simar, L. and P. W. Wilson (2000). Statistical inference in nonparametric frontier models: The state of the art. Journal of productivity analysis 13(1), 49-78.

Triebel, H. (1994). Theory of function spaces ii. Bull. Amer. Math. Soc 31, 119-125.

Tsybakov, A. B. (2009). Introduction to nonparametric estimation. revised and extended from the 2004 french original. translated by vladimir zaiats. 